\title{
CDISC SDTM Biospecimen Characteristics Test Name Terminology
}

National Cancer Institute

\section{Source}

National Cancer Institute. CDISC SDTM Biospecimen Characteristics Test Name

Terminology. NCI Thesaurus. Code C124299.

Terminology associated with the biospecimen characteristics test name codelist of the Clinical Data Interchange Standards Consortium (CDISC) Study Data Tabulation Model (SDTM). 